\title{
ANÁLISE DA INFLUÊNCIA DO NISTAGMO ESPONTÂNEO E PRÉ-CALÓRICO NA VECTOELETRONISTAGMOGRAFIA
}

\author{
Analysis of spontaneous and per caloric nistagmus \\ and its influence in vectonystagmography
}

\author{
Gisiane Munaro ${ }^{(1)}$, Pricila Sleifer ${ }^{(2)}$, Fleming Salvador Pedroso ${ }^{(3)}$
}

\begin{abstract}
RESUMO
Objetivo: verificar a ocorrência do nistagmo espontâneo / pré-calórico, sua direção, cálculo da velocidade angular média e real da componente lenta, sua influência quantitativa e qualitativa nas provas calórica e rotatória em pacientes com sintomas vestibulares Métodos: no período de janeiro a abril de 2006, 88 pacientes foram avaliados na Clínica de Audiologia do Centro Diagnóstico Mãe de Deus Center, em Porto Alegre, por Vectoeletronistagmografia computadorizada. Resultados: 33 pacientes (37.5\%) apresentaram nistagmo espontâneo e pré-calórico (olhos fechados), 19 mulheres $(57,6 \%)$ e 14 homens $(42,4 \%)$, idades entre 15 e 81 anos. Foram registradas 31 ocorrências do nistagmo espontâneo e 33 do pré-calórico. A análise estatística demonstrou diferença significante entre as médias da velocidade angular média e real, e entre o nistagmo espontâneo e o pré-calórico. Não houve correlação significativa entre as médias dos nistagmos espontâneo e pré-calórico com os resultados percentuais da prova calórica, porém, foi observada influência qualitativa, pela maior ocorrência de resultados do tipo predomínio direcional. Na prova rotatória, verificou-se assimetria em 11 casos (33,3\%), coincidindo com a direção do nistagmo espontâneo e/ou pré-calórico. Conclusão: uma velocidade angular média de maior valor no nistagmo espontâneo e pré-calórico não determinou uma assimetria quantitativamente maior na prova calórica, embora qualitativamente observou-se uma maior ocorrência de predomínio direcional do nistagmo para o mesmo lado do nistagmo espontâneo e pré-calórico.
\end{abstract}

DESCRITORES: Eletronistagmografia; Nistagmo Patológico; Testes de Função Vestibular

\section{INTRODUÇÃO}

Movimentos oculares fisiológicos ou induzidos por estimulações visuais representam o elemento principal na investigação e avaliação dos distúrbios vestibulares, sendo a vectoeletronistagmografia (VENG) o instrumento de pesquisa dos movimentos oculares direta ou indiretamente relacionados com a função vestibular ${ }^{1}$. Por análise computadorizada,

(1) Fonoaudióloga do Centro Clínico Mãe de Deus Center, Porto Alegre, RS; Mestranda em Distúrbios da Comunicação Humana pela Universidade Federal de Santa Maria.

(2) Fonoaudióloga do Hospital de Clínicas de Porto Alegre, RS; Docente do Centro Universitário Metodista do Sul, IPA, Porto Alegre, RS; Mestre em Ciências Médicas: Pediatria pela Universidade Federal do Rio Grande do Sul.

(3) Médico Neurologista; Docente do Centro Universitário Metodista do Sul, IPA, Porto Alegre, RS; Doutor em medicina pela Universidade Federal do Rio Grande do Sul.

Conflito de interesse: INEXISTENTE a VENG possibilita o cálculo da velocidade angular real da componente lenta (VARCL) do nistagmo, ou VACL real, que, juntamente com o registro em três canais, facilita o reconhecimento da direção dos movimentos oculares, por meio do ângulo formado pela componente rápida. Assim, é possível verificar a direção exata, amplitude e VACL média dos batimentos nistágmicos selecionados em cada canal, fornecido pela VARC ${ }^{2}$.

A presença do nistagmo espontâneo pode indicar desequilíbrio entre os labirintos, ocasionado, por exemplo, por uma lesão vestibular aguda. 0 nistagmo ocorre porque o tônus do labirinto intacto está em desacordo com o tônus do labirinto alterado. As características que ajudam a distinguirmos entre um nistagmo periférico de central incluem os efeitos da fixação visual, a configuração da componente lenta e a direção da rotação ocular ${ }^{3}$. Por outro lado, o nistagmo espontâneo com olhos fechados pode ser fisiológico, devido a uma provável incapacidade 
do sistema vestibular em estabilizar por completo a posição dos olhos quando o apoio visual é removido. Em indivíduos normais, pode-se observar nistagmo de direção variada, podendo ser horizontal, vertical ou oblíquo ${ }^{4}$.

Nestes indivíduos, o nistagmo espontâneo pode estar presente com olhos fechados, de direção horizontal e VACL igual ou inferior $6 \% \mathrm{~s}^{6}$ ou até $7 \%$ s. $\mathrm{Na}$ presença do nistagmo espontâneo, as respostas pós-rotatórias podem estar assimétricas, apresentando preponderância direcional para o mesmo lado do nistagmo. Em alguns casos, a influência deste pode causar $100 \%$ de assimetria nesta prova. A prova calórica também é influenciada, embora em menor grau que a prova rotatória pendular decrescente (PRPD), pela presença de nistagmo espontâneo e, como consequência, pode surgir uma assimetria direcional. A avaliação quantitativa do nistagmo é realizada pelo cálculo da VACL, que exprime o número de graus de desvio angular dos olhos, onde cada milímetro de altura (ou de desvio ocular) equivale a $1^{\circ}$, cuja medida final é expressa em graus por segundo ${ }^{5}$. Com relação ao nistagmo espontâneo, embora uma VACL de até $7 \%$ s seja considerada como valor de normalidade, quando presente, tanto em pessoas normais como em pessoas com sintomas vestibulares, varia entre $1 \%$ a $5 \%$ s. Os valores acima de $7 \%$ são observados em pacientes que sofreram crise labiríntica recente ${ }^{6}$.

O reflexo vestíbulo-ocular consiste na transformação de um sinal sensorial vindo dos canais semicirculares em uma resposta motora ocular, auxilia para estabilizar o olhar durante os movimentos cefálicos. Os movimentos oculares reflexos ocorrem na direção oposta à rotação cefálica e de velocidade equivalente a esta ${ }^{7,8}$. Há uma correlação entre os reflexos vestibulares e a faixa etária, ou seja, ocorre declínio do reflexo vestíbulo-ocular com o aumento da idade, em contrapartida, há um aumento do reflexo cérvico-ocular para compensar a redução da atividade vestibular ${ }^{9}$. O conhecimento de aspectos detalhados da fisiologia dos reflexos vestibulares é necessário para melhor compreensão dos mecanismos de processamento central que atuam sobre a movimentação ocular reflexa, uma revisão detalhada deste assunto pode ser encontrada em bibliografia específica ${ }^{8}$. Portanto, o nistagmo espontâneo pode resultar dos sinais neurais transportados para os neurônios oculomotores. Um desequilíbrio vestibular produz uma migração constante dos olhos em uma direção, interrompida pelas componentes rápidas na direção oposta. Lesões do sistema vestibular periférico reduzem os sinais aferentes tônicos originados dos receptores de um dos labirintos. A componente horizontal domina, sugerindo que as atividades tônicas dos canais verticais intactos e dos órgãos otolíticos se cancelam parcialmente ${ }^{10}$. O nistagmo espontâneo pode surgir por decréscimo do tônus vestibular unilateralmente, causado por uma lesão periférica ou de núcleos vestibulares, bem como pode ocorrer um predomínio direcional para o lado saudável ${ }^{11}$.

A falta de consenso na interpretação do exame quando na presença de nistagmo espontâneo e pré-calórico estimulou o interesse em pesquisar sua influência nas provas calóricas da VENG computadorizada. Pela prática clínica, observa-se que o nistagmo espontâneo exerce influência em algumas provas do exame, especialmente na PRPD e prova calórica, mas será essa influência proporcional ao grau da VACL desse nistagmo?

Neste estudo, interessa analisar o papel da VENG na presença de nistagmo espontâneo e précalórico, em indivíduos com queixas vestibulares e/ou auditivas. Buscou-se confrontar os valores da VARCL, bem como da VACL média do nistagmo espontâneo / pré-calórico com o pós-calórico, a fim de obter informações que possam contribuir para a avaliação e diagnóstico otoneurológico. Um consenso a respeito da ocorrência e interpretação desses aspectos na patologia vestibular é indispensável para a compreensão do seu significado nos distúrbios do equilíbrio.

O presente estudo tem como objetivo verificar a ocorrência do nistagmo espontâneo e pré-calórico em pacientes com sintomas vestibulares, analisando sua direção, grau da velocidade angular média e real da componente lenta, bem como sua influência quantitativa e qualitativa nas provas calórica e rotatória.

\section{MÉTODOS}

Estudo de caráter transversal, observacional, retrospectivo, individual, não comparado e contemporâneo, realizado no Setor de Audiologia do Centro Clínico Mãe de Deus, na cidade de Porto Alegre, RS, entre janeiro a abril de 2006. Foram incluídos na pesquisa todos os pacientes sintomáticos que apresentaram nistagmo espontâneo e/ou pré-calórico com olhos fechados na VENG.

Foi realizada anamnese para registro de dados sobre saúde atual e pregressa. A obtenção dos registros vestíbulo-oculares com eletrodos foi realizada pelo sistema computadorizado de vectoeletronistagmografia - Vecwin Digital versão 5.0, da Neurograff. As análises foram feitas a partir de trechos selecionados pela examinadora, que correspondeu à maior reposta nistágmica de cada prova.

A pesquisa do nistagmo espontâneo é realizada com o paciente sentado, no olhar frontal, com olhos abertos e fechados. Quando o paciente está 
deitado de costas, na posição em que serão realizadas as provas calóricas (posição I de Brünings), é pesquisado o nistagmo pré-calórico. Estes, quando presentes, podem influenciar as respostas póscalóricas de mesma direção, resultando em um predomínio direcional (PDN) ${ }^{6}$. O registro do nistagmo espontâneo com olhos fechados foi de 80 segundos para cada paciente, sendo o registro do nistagmo pré-calórico sempre superior ao primeiro. A presença de nistagmo foi considerada quando na existência de, pelo menos, três batimentos de mesma direção com, pelo menos, duas repetições posteriores ${ }^{6}$. Evitaram-se os registros com interferência de artefatos como piscadas, para descartar a possibilidade de o programa calcular nistagmos com uma VACL de valor maior.

A prova rotatória foi realizada com o paciente sentado, olhos fechados, cabeça fletida $30^{\circ}$ para estimulação dos canais semicirculares laterais. A prova calórica consistiu em estimulação com ar $\left(42^{\circ}\right.$ e $\left.18^{\circ}\right)$ em ambas as orelhas durante 80 segundos, com intervalo de 5 minutos entre as estimulações. Foram realizadas tarefas de alerta mental durante todas as provas com olhos fechados a fim de maximizar as respostas do reflexo vestíbulo-ocular, pois o reflexo vestíbulo-ocular pode ser suprimido por fadiga ou desatenção ${ }^{12}$.

A redução nas respostas de um labirinto, em comparação com o outro, geralmente é demonstrada por uma paresia nas respostas calóricas. No presente estudo, os valores de normalidade para um predomínio labiríntico (PL) situaram-se até o limite de 33\%, obtido pela fórmula de Jongkees. Um PDN indica um nistagmo de maior amplitude em uma direção do que na outra, determinada pelos valores de normalidade que aqui se limitaram em até $22 \%$. Para valores absolutos, estão dentro da normalidade a VACL média entre 2 a $19 \%$ s, representando uma hiperreflexia se acima do último, ou uma hiporreflexia se inferior ao primeiro. Estes valores variam conforme o tipo de estímulo (água ou ar) e com as especificações de cada equipamento e foram estabelecidos como referência, a partir de estudo realizado para padronização ${ }^{13}$. As provas calórica e rotatória são complementares, sendo que a primeira depende da transferência de energia térmica através da orelha e sofre influência das variações de tamanho da orelha externa, o que pode causar grande variabilidade entre sujeitos ${ }^{12}$.

A realização deste estudo foi aprovada através de projeto apresentado ao Comitê de Ética do Centro Universitário Metodista - IPA, registro $n^{\circ} 1498$ e pelo Comitê de Ética do Hospital Mãe de Deus, autorizando a utilização do banco de dados do Setor de Audiologia do Mãe de Deus Center.

O programa computadorizado de VENG realizou a análise automática dos trechos selecionados, fornecendo os valores da VACL média e real das provas pertinentes ao estudo. As análises estatísticas foram feitas através do programa SPSS 13.0. Para comparação entre as médias da VACL média e real, foi utilizado o teste "t" de Student, para os valores dos nistagmos espontâneo e pré-calórico. Para avaliar a correlação entre a VACL média dos nistagmos espontâneo e pré-calórico e os valores para PDN e PL da prova calórica, foi utilizado o coeficiente de Pearson ao nível de significância de $0,5 \%$.

\section{RESULTADOS}

Dos 88 pacientes que realizaram avaliação otoneurológica no período estabelecido, 33 (37,5\%) apresentaram nistagmo espontâneo e/ou précalórico na pesquisa com olhos fechados. A faixa etária variou entre 15 e 81 anos, média de idade 43,9 anos, desvio padrão $\pm 18,18$, sendo $19(57,6 \%)$ indivíduos do sexo feminino e 14 (42,4\%) do sexo masculino.

Conforme a Tabela 1 , foram registradas 31 ocorrências de nistagmo espontâneo, com valores de VACL média que variaram entre $2,1 \%$ e $6,9 \%$, com média de $3,61 \%$. A VARCL variou entre $2,2 \%$ s e $9,3 \%$, com média de $4,42 \%$. A presença de nistagmo pré-calórico foi registrada em 33 casos, com valores de VACL média entre $2,5 \%$ s e $10 \%$,

Tabela 1 - Valores para VACL média e real do nistagmo espontâneo e pré-calórico

\begin{tabular}{lccccc}
\hline & $\mathbf{n}$ & VACL mín. $(\% / \mathbf{s})$ & VACL máx. $(\% / \mathbf{s})$ & Média (\%) & Desvio Padrão \\
\hline Ny Esp VACL & 31 & 2,10 & 6,90 & 3,61 & $\pm 1,06$ \\
Ny Esp VARCL & 31 & 2,20 & 9,30 & 4,42 & $\pm 1,78$ \\
Ny Pré VACL & 33 & 2,50 & 10,0 & 4,11 & $\pm 1,66$ \\
Ny Pré VARCL & 33 & 2,70 & 12,10 & 4,98 & $\pm 2,48$ \\
\hline
\end{tabular}

${ }^{*}$ Ny Esp - nistagmo espontâeno

*Ny Pré - nistagmo pré-calórico

*VACL - velocidade angular média da componente lenta

*VARCL - velocidade angular real da componente lenta 


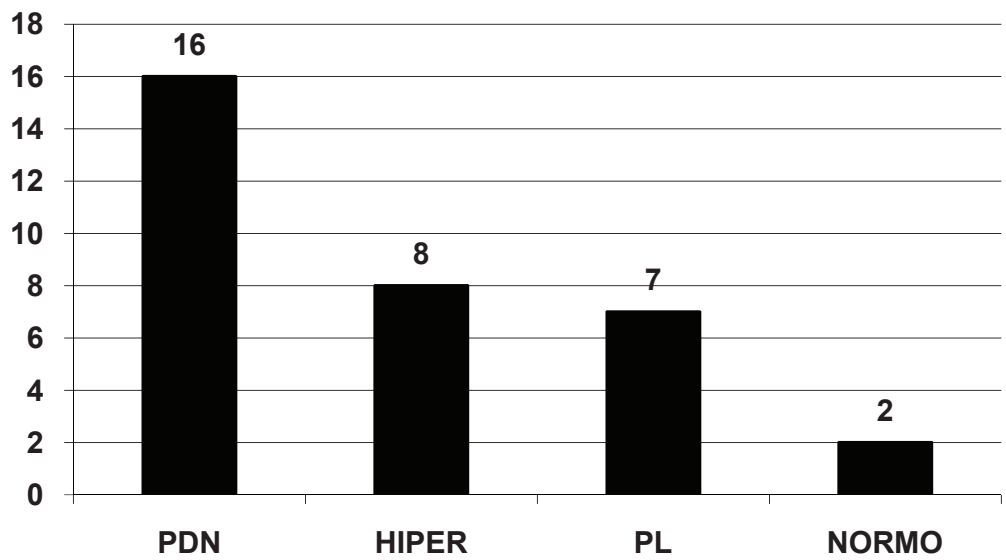

*PDN: Predomínio Direcional do Nistagmo ${ }^{*}$ HIPER: Hiperreflexia *PL: Predomínio Labiríntico *NORMO: Normorreflexia

\section{Figura 1 - Resultados da prova calórica}

média de $4,11 \%$ s e valores de VARCL entre $2,7^{\circ} / \mathrm{s} \mathrm{e}$ $12,1 \%$, média de $4,98 \%$ s.

Os resultados da prova calórica evidenciaram maior ocorrência de PDN com 16 casos (45,4\%), seguido por hiperreflexia em oito casos $(24,2 \%), \mathrm{PL}$ em sete $(21,2 \%)$ e normorreflexia em dois $(6,06 \%)$, conforme a Figura 1. Ocorreram quatro casos de hiperreflexia unilateral e quatro de hiperreflexia bilateral. A normorreflexia em dois casos ocorreu associada a alterações nas provas de posição, com características típicas de vertigem posicional paroxística benigna (VPPB).

Quando feita a correlação de Pearson entre os valores da VACL média e os resultados percentuais da prova calórica, verificou-se que não houve correlação entre o grau da VACL dos nistagmos espontâneo e pré-calórico com os percentuais encontrados para PDN e PL, sendo os valores de p: para a correlação entre nistagmo espontâneo e PDN, $p=0,833$, entre nistagmo pré-calórico e PND, $p=0,336$; para as correlações entre nistagmo espontâneo e $P L, p=0,660$ e entre nistagmo pré-calórico e PL, $p=0,808$. Verificou-se assimetria da PRPD em 11 casos $(33,3 \%)$, sugerindo menor influência do nistagmo sobre esta prova, quando comparado à prova calórica.

Com relação à direção do nistagmo, foram encontrados 14 (42,4\%) registros do tipo horizontal, sendo seis horizontais para a direita e oito horizontais para a esquerda. Do tipo oblíquo, somaram 19 casos $(57,5 \%)$, sendo seis oblíquos para direita e para cima e três para baixo; e dois oblíquos para a esquerda e para cima e oito para baixo. Não foi encontrada nenhuma ocorrência de nistagmo espontâneo ou pré-calórico de direção vertical. A direção dos nistagmos espontâneo e pré-calórico sempre coincidiu, não houve variações de sentido entre eles, ou seja, nistagmo oblíquo para direita e para cima eliciados no registro do nistagmo espontâneo foi confirmado no pré-calórico.

Tabela 2 - Direção do nistagmo espontâneo e pré-calórico

\begin{tabular}{lcc}
\hline Direção nistágmica & Ocorrências & $\%$ \\
\hline Horizontal para Direita & 6 & 18,18 \\
Horizontal para Esquerda & 8 & 24,24 \\
Oblíquo p/direita e p/cima & 6 & 18,18 \\
Oblíquo p/esquerda e p/cima & 2 & 6,06 \\
Oblíquo p/direita e p/baixo & 3 & 9,09 \\
Oblíquo p/esquerda e p/baixo & 8 & 24,24 \\
Total & 33 & 100 \\
\hline
\end{tabular}




\section{DISCUSSÃO}

Em pesquisa com indivíduos normais ${ }^{4}$, a presença do nistagmo espontâneo foi atribuída à sensibilidade do equipamento utilizado e à análise subjetiva do exame, visto que as alterações de ritmo e morfologia do traçado podem dificultar a definição da presença do nistagmo. A VENG computadorizada possibilita o registro de rotações oculares mínimas a partir de $0.5 \%$, embora a presença de artefatos musculares, especialmente em idosos, limite essa sensibilidade para $1^{\circ}$ ou $2^{\circ}{ }^{10}$. No presente estudo, a média dos valores nistágmicos mínimos registrados foram VACL de $2,1 \%$ e máximas de $2,1 \%$ s e $6,9 \%$, enquanto estudo com indivíduos normais encontrou limites inferior e superior de VACL média para o nistagmo espontâneo igual a $1,0 \%$ e $4,5 \%$ $s$ respectivamente, sendo a média aritmética de $2,3^{\circ} / \mathrm{s}^{14}$, inferior à encontrada no presente estudo com indivíduos sintomáticos, que foi de $3,6 \%$ s.

Os mesmos autores, quanto à direção, verificaram presença do nistagmo espontâneo com olhos fechados horizontal em quatro $(80,0 \%)$ dos cinco indivíduos normais em que foi encontrado e oblíquo em um $(20,0 \%)$ caso. Em outro estudo com indivíduos normais, a direção predominante também foi a oblíqua, porém, a de menor ocorrência foi a horizontal, embora os autores encontraram alta variabilidade para este parâmetro ${ }^{4}$. A direção do nistagmo é definida pela observação dos três canais de registro: um nistagmo de direção horizontal tem o mesmo sentido nos dois primeiros canais e sentido oposto no terceiro, um nistagmo vertical tem amplitude reduzida no primeiro e mesmo sentido nos demais canais, já o nistagmo oblíquo tem mesmo sentido nos dois primeiros e está reduzido no terceiro canal, ou ainda, apresenta sentidos opostos no primeiro e terceiro e encontra-se reduzido no segundo canal ${ }^{15}$.

Dados americanos demonstram que $20 \%$ dos indivíduos normais têm nistagmo espontâneo e $75 \%$ têm nistagmo posicional, quando testados com olhos fechados, ou abertos no escuro. Na prática clínica, o nistagmo espontâneo ou posicional que exceder o valor de $4 \%$ s é considerado um sinal de comprometimento vestibular ${ }^{10}$. Outros autores consideram anormal a presença do nistagmo espontâneo quando a VACL exceder o valor de $5^{\circ} / \mathrm{s}^{16}$. No presente estudo, a porcentagem de indivíduos sintomáticos que apresentaram nistagmo espontâneo foi de 37,5\%.

Em indivíduos normais, este fenômeno ocular é de ocorrência rara, geralmente surge com olhos fechados e de VACL inferior a $7 \%$ s na eletronistagmografia e a $10 \%$ na VENG. Em patologias vestibulares periféricas, no intervalo entre as crises vertiginosas ou em casos que não apresentam episódios de vertigem aguda, pode ocorrer nistagmo espontâneo somente com olhos fechados, de VACL superior à presente em indivíduos normais ${ }^{17}$.

Por não haver consenso nos valores de VACL do nistagmo espontâneo e pré-calórico, nessa casuística, foram considerados os nistagmos de qualquer valor, sendo que VACL de $1 \%$ a $5 \%$ s podem ser observados em indivíduos assintomáticos ou com queixas ${ }^{6}$. Em indivíduos com vestibulopatias periféricas crônicas, pode aparecer nistagmo com velocidade inferior, igual ou superior a $7^{\circ} / \mathrm{s}^{1}$.

$O$ teste " $t$ " de Student demonstrou diferença significante entre as médias realizadas pela VACL média e real, ou seja, a VARCL foi maior que a VACL média tanto nos registros do nistagmo espontâneo quanto pré-calórico, pois esta representa a média das VACL considerando os três canais de registro, possibilitando a obtenção da direção exata, amplitude e VACL dos batimentos nistágmicos selecionados em cada canal ${ }^{2}$.

Ao observar os valores médios para VACL do nistagmo espontâneo e pré-calórico, verificou-se que houve diferença estatisticamente significante entre os mesmos $(p=0,004)$, sendo que o pré-calórico apresentou valores mais altos. Entretanto, quando verificados considerando a VARCL do nistagmo espontâneo e pré-calórico, essa diferença não foi significante.

Foi observado que a presença isolada do nistagmo pré-calórico ocorreu em dois casos, porém, o nistagmo espontâneo ocorreu sempre acompanhado do pré-calórico. É possível que, a simples mudança da posição da cabeça provoque o aparecimento do nistagmo. Por isso, é indispensável pesquisá-lo antes das provas calóricas e rotatórias ${ }^{6}$.

Os resultados da prova calórica demonstraram que o PDN foi o achado de maior ocorrência, sempre de mesmo sentido do nistagmo espontâneo e/ ou pré-calórico, conforme esperado ${ }^{6}$ e sugerindo um reforço do mesmo, embora o teste coeficiente de Pearson demonstrou ausência de correlação entre os valores de VACL média encontrada nos nistagmo espontâneos e pré-calóricos e os valores percentuais para PDN e PL. O PL foi observado sempre do lado em que apareceu o nistagmo espontâneo e pré-calórico, ou seja, os nistagmos batendo para o lado esquerdo indicam o lado está saudável, sugerindo que o lado oposto encontrase deficitário ${ }^{11}$. Observa-se, portanto, que as provas calóricas sofrem influência destes nistagmos mesmo quando são de pequena VACL e devem ser considerados na análise do exame. Sugere-se para que os valores de normalidade sejam discutidos a partir de mais estudos a respeito.

Portanto, um nistagmo espontâneo e pré-calórico de maior valor não necessariamente determinou um predomínio direcional ou labiríntico maior do 
que nistagmos de menor valor, quando verificados a partir dos valores da VACL média. Os valores da VARCL não foram correlacionados, pois, os resultados percentuais da prova calórica foram fornecidos pelo programa considerando somente a análise da VACL média, não fazendo sentido, portanto, analisá-los pela VARCL. A ocorrência de hiperreflexia foi o segundo resultado mais observado, sempre nas provas frias, com o mesmo número de ocorrências unilateral e bilateral. Deve-se lembrar, que as provas frias têm como resultado, nistagmos em direção contrárias à orelha estimulada, ou seja, na prova fria da orelha direita, espera-se encontrar nistagmos batendo para a esquerda, e vice-versa ${ }^{5}$.

A ocorrência de normorreflexia em dois indivíduos demonstrou ausência de influência, ao menos qualitativa, dos nistagmos espontâneo e pré-calórico, nestes casos. Porém, a ocorrência dos mesmos foi concomitante a alterações nas provas de posição, sugestivas de um quadro de VPPB, onde a normorreflexia é um achado comum nesses casos, e o diagnóstico é baseado nos sinais observados e sintomas referidos nas provas de posição ${ }^{18,19}$.

A presença de assimetria na PRPD foi observada em $33,3 \%$ dos casos, com predomínio direcional sempre no mesmo sentido do nistagmo espontâneo e/ou pré-calórico. Os achados deste estudo sugerem que esta prova sofreu menor influência destes fenômenos do que a prova calórica, discordando de autores ${ }^{5}$ e aproximando-se do estudo em que verificaram comportamento similar à PRPD e à prova calórica ao correlacionar a VARCL máxima de ambas as provas ${ }^{15}$. Sugere-se que mais pesqui- sas sejam realizadas neste sentido, considerando a carência de estudos específicos a respeito do nistagmo espontâneo e pré-calórico, mesmo porque, os resultados do presente estudo vão de encontro às referências disponíveis.

\section{CONCLUSÃO}

Diante do que foi possível observar na VENG sobre a presença do nistagmo espontâneo/précalórico, a prova calórica e a PRPD, constata-se, através das análises estatísticas, diferença significante entre as médias da VACL média e real, sendo a última maior que a primeira, tanto para o nistagmo espontâneo quanto para o pré-calórico; diferença significante entre os valores encontrados para o nistagmo espontâneo e o pré-calórico quando analisados pela VACL média, sendo o précalórico de maior valor. Entretanto, esta diferença não ocorre quando analisados pela VARCL. Ainda, não se observa diferença significante entre as correlações realizadas para as VACL média e real e os resultados quantitativos das provas calóricas (PDN e $\mathrm{PL})$, porém, há influência qualitativa observada, principalmente, pela maior ocorrência de resultados do tipo PDN.

Pela observação qualitativa, verifica-se que a direção predominante dos nistagmos espontâneo e pré-calórico foi a oblíqua, seguida pela horizontal; a assimetria na PRPD na presença dessas manifestações oculares ocorre em menos casos do que o predomínio direcional do nistagmo, na prova calórica.

\begin{abstract}
Purpose: to check the behavior of spontaneous and per caloric nystagmus, its occurrence, direction and its quantitative and qualitative influence in the caloric and rotatory testing in symptomatic subjects. Methods: from January to April 2006, 88 patients were evaluated at Mãe de Deus Center, Porto Alegre city, through Vectonystagmography digital. Results: $33(37.5 \%)$ subjects showed spontaneous and per caloric nystagmus with eyes closed, 19 female (57.6\%), 14 male (42.4\%), ages between 15 and 81 years old. 31 occurrences of spontaneous nystagmus and 33 of per caloric nystagmus were recorded. The main direction of the nystamus was oblique, followed by horizontal, as expected. The statistic analysis through SPSS 13.0 program showed there were significant differences between the spontaneous and per caloric nystagmus comparing the angular velocity of slow component mean value, being the per caloric higher than the spontaneous. No correlation was found between the slow component mean value of spontaneous and per caloric nystagmus when compared to percentage results of caloric testing, otherwise, qualitative influence was observed because the great occurrence of directional preponderance. Rotational chair testing asymmetry was verified in only 11 (33.3\%) patients. Conclusion: the statistic analysis of mean values showed no significant correlations among the angular velocity of slow component of the nystagmus spontaneous and per caloric compared with the of caloric testing percentages.
\end{abstract}

KEYWORDS: Electronystagmography; Nystagmus, Pathologic; Vestibular Function Tests 


\section{REFERÊNCIAS}

1. Ganança MM, Caovilla HH, Munhoz MSL, Silva MLG, Frazza MM. As etapas da equilibriometria. In: Caovilla HH, Ganança MM, Munhoz MSL, Silva MLG. Equilibriometria clínica. São Paulo: Atheneu; 1999. p. 41-114.

2. Ito YI, Ganança MM, Caovilla HH, Albernaz PLM. Vecto-electronistagmografia: evolução. RBM-ORL. 1994; 9(1):49-53.

3. Walker MF, Zee DS. Bedside vestibular examination. Otolaryngol Clin North Am. 2000; 33(3):495-506.

4. Alvarenga KF, Lopes ES, Michelli ACD, Ito YI, Caovilla HH, Ganança MM. Da ocorrência e direção do nistagmo espontâneo em indivíduos normais à vecto-eletronistagmografia. Acta AWHO. 1995; 14(1):14-7.

5. Mangabeira Albernaz PL, Ganança MM. Atlas de electronistagmografia. São Paulo: Editamed; 1977.

6. Mor R, Fragoso M, Taguchi CK, Figueiredo JFFR. Vestibulometria e fonoaudiologia: como realizar e interpretar. São Paulo: Lovise; 2001.

7. Broussard DM, Kassardjian CD. Learning in a simple motor system. Learn Memory. 2004; 11(2):127-36.

8. Angelaki DE. Eyes on target: what neurons must do for the vestibuloocular reflex during linear motion. J Neurophysiol. 2004; 92(1):20-35.

9. Kelders WPA, Kleinrensink GJ, Van der Geest JN, Feenstra L, Zeeuw CI, Frens MA. Compensatory increase of the cervico-ocular reflex with age in healthy humans. J Physiol. 2003; 553(1):311-7.

10. Honrubia V. Testes quantitativos da função vestibular e o exame clínico. In: Herdman SJ. Reabilitação vestibular. São Paulo: Manole; 2002. p.105-68.
11. Carvajal IW. Directional preponderance of the post-caloric nystagmus. Acta AWHO. 1987; 6(2):102-3.

12. Fife TD, Tusa RJ, Furman JM, Zee DS, Frohman E, Baloh RW, et al. Assessment: vestibular testing techniques in adults and children: report of therapeutics and technology assessment subcommittee of the American Academy of Neurology. Neurology. 2000; 55(10):1431-41.

13. Ganança CF, Souza JAC, Segatin LA, Caovilla HH, Ganança MM. Limites normais dos parâmetros de avaliação à vectonistagmografia digital Neurograff. Acta AWHO. 2000; 19(2):105.

14. Braga HM, Ito YI, Falsetti HC, Caovilla HH, Novo $\mathrm{NF}$, Ganança MM, et al. Nistagmo espontâneo e semi-espontâneo a vecto-electronistagmografia em individuos normais. Rev Bras Otorrinolaringol. 1981; 47(2):127-40.

15. Queiroz BMA, Ito $\mathrm{YI}$, Falsetti HDC, Caovilla HH, Ganança MM, Albernaz PLM. Da comparação entre a prova rotatória pendular crescente e a prova calórica, em termos de velocidade angular real máxima da componente lenta do nistagmo em indivíduos normais. Acta AWHO. 1984; 3(1):19-27. 16. Ruckenstein MJ, Shepard NT. Balance function testing: a rational approach. Otolaryngol Clin North Am. 2000; 33(3):507-18.

17. Caovilla HH, Ganança MM. Equilibriometria. In: Ganança MM, Vieira RM, Caovilla HH. Princípios de otoneurologia. São Paulo: Atheneu; 1998.

18. Koga KA, Resende BDA, Mor R. Estudo da prevalência de tonturas/vertigens e das alterações vestibulares relacionadas à mudança de posição de cabeça por meio da vectoeletronistagmografia computadorizada. Rev CEFAC. 2004; 6(2): 197-202.

19. Tirelli G, D'Orlando E, Giacomarra V, Russolo M. Benign positional vertigo without detectable nystagmus. Laryngoscope. 2001; 111(6):1053-6.

RECEBIDO EM: 03/09/2007

ACEITO EM: 06/04/2009

Endereço para correspondência:

Gisiane Munaro

Av. Francisco Trein, 487/603

Porto Alegre - RS

CEP: $91350-200$

E-mail: gisiane_munaro@yahoo.com.br 